\title{
A NOTE ON CREDIT INSURANCE
}

\author{
BY \\ JOHANNES LEITNER
}

\begin{abstract}
In a simple stationary setting with constant interest rate, we derive pricing formulas for defaultable bonds with stochastic recovery rate using a replication argument. Replication is done by using an insurance contract (i.e. a kind of credit default swap), the price of which is determined by a dynamic premium calculation principle. We consider two cases, a linear one, where pricing amounts to solving an inhomogeneous linear ODE, and a super-linear case where a Riccati ODE has to be solved.
\end{abstract}

\section{KEYWORDS}

Credit insurance, credit default swaps, random measures, term structure for defaultable bonds, dynamic premium calculation principle.

\section{INTRODUCTION}

On an abstract level insurance markets and financial markets are quite similar. Both types of markets can be modeled in a semimartingale setting and a noarbitrage property (no-free lunch with vanishing risk) can then be characterized by the existence of an equivalent martingale measure (EMM), see Delbaen and Schachermayer (1994). Especially if price processes are not continuous, EMMs are typically not unique (if they exist at all). In order to price a nonattainable contingent claim, say a non-deterministic payment $Y$ at some terminal (stopping) time $T$, usually an EMM $Q$ is chosen and the price process for the claim $Y$ is defined by $E_{Q}\left[Y \mid \mathcal{F}_{t}\right], t \geq 0$. This method allows to extend an existing market without creating arbitrage opportunities. The problem is of course how to choose the EMM $Q$. The fact that one actually has to choose an EMM without having a canonical way how to do this, is often hidden by saying something like 'working under risk-neutral probabilities...'. One way is to choose an EMM $Q$ such that it optimizes some criteria, e.g. such that $\left\|\frac{d Q}{d P}\right\|_{q}$ resp. its relative entropy w.r. to the objective probability measure $P$ is minimal, see Delbaen et al. (1997) and the references therein, resp. Frittelli (2000) and Delbaen et al. (2002). A related, well-known and extensively studied way is to 
consider an expected utility maximization problem and choose $Q$ as a solution for the corresponding dual optimization problem, see e.g. Kramkov and Schachermayer (1999) and Schachermayer (1999). In applications one often chooses a parameterized class of probability measures equivalent to $P$ and determines parameters by fitting prices calculated under the implied EMM to prices observed in the market. This approach bears the dangers of making an incomplete market look like a complete one: Having sold a non-attainable derivative at such an implied price, there is no guarantee that there is a good way to hedge the resulting risk!

In any case, there seems not to be a generally agreed on way of choosing an EMM for pricing in a non-complete market setting. Even worse, given the enormous size of the set of all possible derivatives one can not expect to establish a liquid market for all derivatives. This does not exclude that for any single derivative one can find someone who is willing to sell it as an OTC deal. In this setting prices might be found applying (utility) indifference arguments. They will then in general depend on the sellers portfolio and will not be linear in the payoff. This situation is actually very much the situation one faces in a real-world insurance market, where e.g. insurance policies can typically not be traded and contacts might be irreversible (at least for some period of time). Possibly for this reason the illusion that an insurance market could be complete never came up and to our knowledge there does not exist a generally accepted way for pricing insurance contracts. On the other hand, insurance contracts are a very natural way to extend a non-complete market and price non-hedgeable risks. Premium calculation principles have been intensively studied (see e.g. Goovaerts et al. (2003) and references therein), but the focus of actuarial mathematics seems to have rather been the study of the probabilities of ruin given some insurance premium.

The purpose of this paper is twofold. Firstly, we introduce dynamic premium calculation principles (DPCPs). Essentially, we propose to split up a classical insurance contract running over a certain period of time into a stream of insurance contracts covering a risk over an infinitesimal time interval. These contracts will be priced by using an instantaneous version of the classical premium calculation principles. We have little to say about which principle is the 'best' one, the advantage of our approach is rather that it allows the buyer of such contracts to exactly replicate in a self-financing way certain contingent claims. (Self-financing here means (as it always does) just that all payments made or received are taken into account in the involved cumulative cash-stream processes). To be more precise, for certain contingent claims (e.g. defaultable credits) with cumulative cash-stream $\left(Z_{t}^{C}\right)_{t \in[0, T]}, 0<T<\infty$, we will replicate $-Z_{t}^{C}$ in a selffinancing way with vanishing initial value, using instantaneous insurance contracts with cumulative cash-stream $\left(Z_{t}^{I}\right)_{t \in[0, T]}$. The insurance contracts then exactly off-set $Z_{t}^{C}$ and the total cumulated cash-stream $Z^{C}+Z^{I}$ vanishes at time $T$, i.e. $Z_{T}^{I}=-Z_{T}^{C}$. In other words, at time $T$ we are exactly in the same situation as if no transaction had been made at all. This off-setting condition determines a relation between the parameters (risk premiums) of the insurance 
prices and $Z_{0}^{C}$. Given the insurance prices there will then exist a unique $Z_{0}^{C}$ such that exact off-setting of $Z_{T}^{C}$ becomes possible. $Z_{0}^{C}$ is then a risk-free price for the cumulated cash stream $Z^{C}$ over $(0, T]$, completely independent of any risk preferences an investor might have.

Using premium calculation principles for pricing has the advantage over risk-neutral pricing that it allows to take into account more explicitly the risk of (extreme) down-side outcomes. The price for this is of course in general non-linearity of the valuation principle. DPCPs take down-side risk into account while at the same time allowing in some important cases for replication of contingent claims! The main idea of a DPCP is to split up the classical security loading of insurance mathematics into a part that compensates for the risk that an insurance claim occurs and a second part for the risk immanent in the claim size given that an insured event occurred at an instant of time. We allow the conditional claim sizes to be time dependent and the second part of the security loading will then in general vary over time as well.

This method will facilitate the pricing of OTC insurance contracts, where there is little or no diversification possible by selling many similar contracts for independent risks. We belief that in a realistic situation it is not possible to define the 'true' or 'fair' value of a derivative, there exist just too many plausible choices for representing an investors preferences and risk aversions. Offering a self-financing method, using a DPCP-priced stream of insurances, that allows to exactly off-set at terminal time $T$ the cumulated cash-stream generated by a contingent claim up to time $T$, results into a completely risk-free (except for default risk of the insurer) and therefore preference-independent way of pricing in the extended market, i.e. relative to the prices of instantaneous insurance contracts!

We hope that this approach will help the unification of financial and insurance markets, which we think is only possible with a non-linear valuation principle.

Secondly, as an explicit example where our approach can be applied, we consider credit insurance contracts, i.e. a type of credit default-swap (see e.g. Bielecki and Rutkowski (2002)), in order to price defaultable bonds in continuous time. Credit default swaps differ form our insurance contracts only insofar as usually payments are due at discrete points in time whereas we use a continuous stream of payments. Furthermore, in our approach the insurance payment in the case of default will include the cost of insurance up to default time! Exact off-setting with discrete payments and insurance payments of only notional minus recovery value of the credit will be much harder to achieve. However, our point is rather that we propose a new pricing rational that replaces the problem of having to choose an EMM from a very large set in order to price the swap by the much simpler problem of choosing a DPCP. (E.g. for vanishing interest rate and coupon, the default probability of the credit w.r.t. to an EMM can be any value in $(0,1)$, i.e. any price strictly between zero and the notional of the credit is compatible with the no-arbitrage criterion.) In other words, if one wants to do better than just fitting some parameterized EMM to observed (e.g. credit default swap-) market prices (if such a liquid 
market exists in the first place), and use this fitted EMM for pricing of nonattainable contingent claims, one way is to introduce new assets to the market. Instead of extending the market by directly introducing credit default swaps, we argue that introducing instantaneous insurance contracts is a good way to enlarge a market in such a way that defaultable credits can be uniquely priced. Insurance companies should be willing to offer such contracts for a large enough risk premium, i.e. an appropriate DPCP. Even if instantaneous insurance contracts are not actually traded, it is still possible to compare would-be prices with observed prices and to determine which derivatives are relatively expensive compared to others.

The self-financing off-setting argument based on DPCPs leads to explicit formulas in this situation.

Possible applications of this approach are the following:

(1) Capital relief: It might be advantageous for a bank to get a single large credit (partially) off the balance sheet and release risk capital by (partially) insuring the credit, if the cost of providing risk capital exceeds the price of an insurance. The idea is that an appropriate insurance contract can have a strong effect on extreme quantiles of the return distribution of a credit portfolio for a comparatively low insurance premium, while regulatory risk capital requirements and refinancing interest rates, which depend on the banks rating, can make too high quantiles of the return distribution quite costly for a bank.

(2) Contract changes: Assume a customer wants to change the redemption scheme of a credit contract, e.g. change the coupon payments, the maturity time, or the notional by paying off part of the loan before maturity. Our method provides a formula to design a value neutral change of a credit contract.

(3) Implied quantities: By fitting parameters to benchmark loans where market prices are known, implied default probabilities can be calculated. E.g. if for a company a term structure of defaultable bond prices is available, implied default probabilities can be calculated.

In Section 2 DPCPs are introduced. In Section 3 two examples of credit insurance are studied, one for the linear case and one for the super-linear case.

\section{Dynamic Premium Calculation Principles}

A premium calculation principle (PCP) assigns to a bounded random variable, modelling the total claim amount, a real number representing the premium charged for the corresponding insurance contract. Gerber (1979) discusses desirable properties of PCPs in a static setting, Mikosch (2004) considers explicitly their evolution over time. For a unified approach and a recent overview see e.g. Goovaerts et al. (2003). 
Any functional $R: L^{\infty}=L^{\infty}(\Omega, \mathcal{F}, P) \rightarrow \mathbf{R}$ will be called a PCP. Classical examples of PCPs are the following, $\left(X \in L^{\infty}\right)$ :

(1) Net principle: $\mathcal{R}(X)=E[X]$.

(2) Expected value principle: $\mathcal{R}(X)=(1+\lambda) E[X]$, where $\lambda \geq 0$ is a safety loading.

(3) (Semi-) Standard deviation principle: $\mathcal{R}(X)=E[X]+\beta \sqrt{\operatorname{Var}_{(+)}(X)}$, where $\beta \geq 0$ and $\operatorname{Var}_{+}(X):=E\left[(X-E[X])_{+}^{2}\right]$ is the so-called (upper) semi-variance.

Many PCPs have been proposed, see e.g. Wang (1996) for PCPs based on Choquet integrals or distorted probabilities. Other PCPs have been constructed by replacing $\sqrt{\operatorname{Var}(X)}$ in the standard deviation principle with a (coherent) risk measure, see Artzner et al. (1999) for the notion of coherent risk measures. For simplicity we will consider only law-invariant functionals $\mathcal{R}$, i.e. $\mathcal{R}(X)$ depends only on the distribution of $X \in L^{\infty}$ and we set $\mathcal{R}\left(P_{X}\right):=\mathcal{R}(X)$ and $E\left[P_{X}\right]:=E[X]$, where $P_{X}$ denotes the image measure of $P$ under $X$.

Let $\left(T_{i}\right)_{i \geq 0}$ be a non-decreasing sequence of stopping times describing the arrival of insurance claims. We assume $T_{0}=0$ and $T_{i+1}>T_{i}$ on $\left\{T_{i}<\infty\right\}$ for all $i \geq 0$. For simplicity we assume $\lim _{i \rightarrow \infty} T_{i}=\infty$, i.e. explosion is excluded. Denote by $N:=\sum_{i=1}^{\infty} \mathbf{1}_{\left[T_{i}, \infty\right)}$ the corresponding counting process and let $\hat{N}$ denote its predictable compensator. Furthermore, let $\left(X_{i}\right)_{i \geq 1}$ be a sequence of non-negative bounded random variables with $\left\{X_{i}=0\right\}=\left\{T_{i}=\infty\right\}$. $X_{i}$ describes the size of the $i$ th insurance claim occurring at time $T_{i}, i \geq 1$. The total claim amount process $S$ is defined by $S=\sum_{i=1}^{\infty} X_{i} \mathbf{1}_{\left[T_{i}, \infty\right)}$. $\left(T_{i}, X_{i}\right)_{i \geq 1}$ is a marked point process with mark space $\mathbf{R}_{+}$, see e.g. Bremaud and Jacod (1977), Bremaud (1981), Last and Brandt (1995) or Liptser and Shiryaev (2000). The corresponding jump process equals the total claim amount process $S$ which can also be described by the following random measure $\mu$ on $(0, \infty) \times \mathbf{R}_{+}$defined by

$$
\mu(\omega ; d t, d x):=\sum_{n \geq 1} \varepsilon_{\left(T_{n}(\omega), X_{n}(\omega)\right)}(d t, d x) \mathbf{1}_{\left\{T_{n}(\omega)<\infty\right\}}, \quad \omega \in \Omega
$$

where $\varepsilon_{a}$ denotes the Dirac measure located at $a$. Denoting by $\mathbf{x}$ the projection $\Omega \times \mathbf{R}_{+} \times \mathbf{R}_{+} \rightarrow \mathbf{R}_{+}$, we have $\mathbf{x} * \mu=S$. Let $\mathcal{F}_{0}$ be a sub- $\sigma$-algebra of $\mathcal{F}$ and define the filtration $\left(\mathcal{F}_{t}\right)_{t \geq 0}$ as the natural filtration generated by $S$ and $\mathcal{F}_{0}$, i.e. $\mathcal{F}_{t}:=$ $\mathcal{F}_{0} \wedge \sigma\left(S_{s}, 0 \leq s \leq t\right)$ for all $t \geq 0$. Let $\mathcal{P}$ denote the predictable $\sigma$-algebra of $\Omega \times$ $\mathbf{R}_{+}$, generated by all left-continuous $\left(\mathcal{F}_{t}\right)$-adapted processes and let $\mathcal{B}_{+}$denote the Borel $\sigma$-algebra of $\mathbf{R}_{+}$.

Since $\mathbf{R}_{+}$is a Blackwell space, see Jacod and Shiryaev (1987), Chapter II.1, we can find a non-negative kernel $K$ from $\left(\Omega \times \mathbf{R}_{+}, \mathcal{P}\right)$ into $\left(\mathbf{R}_{+}, \mathcal{B}_{+}\right)$, with $K(\omega, t$; $(0, \infty))=1$ for all $(\omega, t) \in \Omega \times \mathbf{R}_{+}$and such that the predictable compensator $\hat{\mu}$ of $\mu$ is given by $\hat{\mu}(\omega ; d t, d x)=K(\omega, t ; d x) d \hat{N}_{t}(\omega)$ for all $(\omega, t) \in \Omega \times \mathbf{R}_{+}$. In general we have $K\left(T_{i}, A\right)=P\left(X_{i} \in A \mid \mathcal{F}_{T_{i}}\right)$ for all $A \in \mathcal{B}_{+}$and $i \geq 1$. In the setting to be used later, where $N$ will be a homogeneous Poisson process, independent of the iid random variables $\left(X_{i}\right)_{i \geq 1}, K(\omega, t, \cdot)$ will simply describe the conditional law of $X_{i}$ given $T_{i}=t$ for all $t>0$ and $i \geq 1$. 
We are going to present a new way to construct PCPs by splitting up the risk of the total claim amount into the risk that a new claim occurs and the risk immanent in the particular claim sizes given that a claim occurred. The idea is very simple: Let $\mathcal{R}$ be a functional that assigns a strictly positive real number to any probability distribution on $(0, \infty)$ and define the following cumulative premium process $V$ (in the interest free setting):

$$
V_{t}:=\int_{0}^{t} \mathcal{R}(K(s, \cdot)) d \hat{N}_{s} .
$$

While e.g. in Mikosch (2004) PCPs are applied to the total claim amount $S_{t}$ which leads to an accumulated premium $V_{t}=\mathcal{R}\left(S_{t}\right)$ (from which an instantaneous premium density can be derived be differentiation), we think it is more natural to charge an instantaneous premium according to (2). In a non-stationary setting the insured might end up paying insurance premiums over a period of time where there is no risk at all, or in some states the premium cash-stream might be quite small even so the risk, conditional on the state, is very high. For an irreversible contract this is of course possible, and for some types of insurance, e.g. health insurance, it might be desirable to have deterministic cash-streams. However, in the case of insurance contracts that can be easily entered and cancelled (by both sides), either the insurer or the insured would try to find a better deal in the market, given the conditional risk. In a non-stationary setting this results into non-deterministic insurance premiums, which means that the insured carries part of the risk due to changes in the distribution of the insured risk. On the other hand premiums would be more transparent since they then depend on the actual risk over short period of time. This is not desirable e.g. for a health insurance, but for OTC insurance contracts this fact will make it easier to offer insurance in the first place, as well as lead to lower prices for buyers that are willing to carry part of the risk.

2.1. Interpretation. More general, consider a marked point process $\left(T_{i}, Y_{i}\right)_{i \geq 1}$ with $E$-valued marks $\left(Y_{i}\right)_{i \geq 1}$ for some Blackwell space $(E, \mathcal{B})$, where $\mathcal{B}$ denotes the Borel $\sigma$-algebra of $E$. A simple insurance event is defined as the event $I_{s, t}^{i, B}:=\left\{\left(T_{i}, Y_{i}\right) \in(s, t] \times B\right\}$ for $0 \leq s<t, B \in \mathcal{B}$ and $i \geq 1$. A simple insurance contract $C_{i}^{H}$ for $I_{s, t}^{i, B}$ is defined by a measurable claim size function $H:(s, t] \times B \rightarrow$ $(0, \infty)$ : In the event $I_{s, t}^{i, B}$ the insurer has to cover the claim $X_{i}:=H\left(T_{i}, Y_{i}\right)$. The infinitesimal analog is as follows: In the event $\left\{\left(T_{i}, Y_{i}\right) \in(t, t+d t] \times(y, y+d y]\right\}$ the insurer has to cover for the claim $x=H(t, y)$. If $\mathcal{R}$ is the expectation operator on $E$ times $1+\lambda$, then the insurer is compensated for taking the risk $H\left(T_{i}, Y_{i}\right) \mathbf{1}_{\left.I_{t, t+d t}^{i, y, y d d}\right]}$ by paying a premium of $H(t, y)(1+\lambda) \alpha_{t}$ over the time interval $(t, t+d t]$, where we assume $\hat{N}$ to be absolutely continuous with respect to Lebesgue measure, i.e. $\hat{N}_{t}=\int_{0}^{t} \alpha_{s} d_{s}$ for a strictly positive predictable process $\alpha$. $\lambda$ can be interpreted as a risk premium for the intensity of the claim arrivals. By an additive superposition of such simple insurance contracts we arrive at (2). However, additive superposition of claims is problematic since it does not take 
the variability in $Y_{i}$ or the size of $H\left(T_{i}, Y_{i}\right)$ into account. Consider first the insurance contract $C_{i}^{H}$ for $H=x \mathbf{1}_{(t, t+d t] \times E}$ that pays $x>0$ currency units in the event $\left\{T_{i} \in(t, t+d t]\right\}$ in return for a premium $x(1+\lambda) \alpha_{t}$ paid over the time interval $(t, t+d t]$. Classical PCPs are often assumed to be translation invariant, i.e. $\mathcal{R}(X+c)=\mathcal{R}(X)+c$ for all $c \in \mathbf{R}$. In our setting it seems more appropriate to let the premium for $C_{i}^{H}$ depend on $x$ in a super-linear way, since for large $x$ the probability of ruin for the insurer (holding only contract $C_{i}^{H}$ ) will be strictly positive, while it will be 0 for small $x$. We propose to compensate the insurer for this type of ruin risk by charging a premium of $\left(x(1+\lambda)+\gamma x^{2}\right) \alpha_{t}$ with $\gamma \geq 0$ over the time interval $(t, t+d t]$ for the contract $C_{i}^{H}$. However, choosing $\mathcal{R}(K(t, \cdot))=E[K(t, \cdot)](1+\lambda)+\gamma(E[K(t, \cdot)])^{2}$ would not take the variability of $K(t, \cdot)$ around its mean into account. We therefore propose the following property for $\mathcal{R}$ : Assume for all $x>0$ that

$$
\mathcal{R}(K(t, \cdot-x))=\mathcal{R}(K(t, \cdot))+x(1+\lambda)+\gamma x^{2},
$$

where $K(t, A-x):=0$ for all $A \in \mathcal{B}_{+}$with $A \subseteq(0, x]$. Plausible examples for dynamic premium calculation principles (DPCPs) are the following:

(1) Dynamic net principle: $\mathcal{R}(K(t, \cdot))=E[K(t, \cdot)],(\lambda=\gamma=0)$.

(2) Dynamic expected value principle: $\mathcal{R}(K(t, \cdot))=(1+\lambda) E[K(t, \cdot)],(\gamma=0)$.

(3) Dynamic (semi-) standard deviation principle: $\mathcal{R}(K(t, \cdot))=(1+\lambda) E[K(t, \cdot)]+$ $\gamma(E[K(t, \cdot)])^{2}+\beta \sqrt{\operatorname{Var}_{(+)}(K(t, \cdot))}$, where $\beta \geq 0$.

Replacing $\sqrt{\operatorname{Var}_{(+)}(K(t, \cdot))}$ by an alternative convex risk measure (see Föllmer and Schied (2002)) a large class of DPCPs can be constructed. More generally, $\mathcal{R}$ could be chosen to be time dependent in a $\mathcal{P}$-measurable way.

2.2. Example. Assume for simplicity that $N$ is a Poisson process with constant intensity $\alpha>0$, so that $\hat{N}_{t}=\alpha t$ for all $t \geq 0$, and that $\mathcal{R}(X)=(1+\lambda) E[X]+$ $\gamma(E[X])^{2}+(1+\lambda) \tilde{R}(X)$ for all $X \in L^{\infty}$, where $\tilde{\mathcal{R}}: L_{+}^{\infty} \rightarrow \mathbf{R}_{+}$is a functional satisfying $\tilde{\mathcal{R}}(X+c)=\tilde{\mathcal{R}}(X)$ and $\mathcal{R}(c)=0$ for all $c \in \mathbf{R}_{+}$. For example, one can use for $\tilde{\mathcal{R}}$ a functional of $(X-E[X])_{+}$, e.g. $\tilde{\mathcal{R}}(X):=\beta\left\|(X-E[X])_{+}\right\|_{p}$ for $X \in L^{\infty}$ with $p \in[1, \infty]$ and $\beta \geq 0$. Note that $\mathcal{R}(X+c)>\mathcal{R}(X)$ holds for all $X \in L^{\infty}$ and constants $c>0$.

\section{Credit Insurance}

For general material on credit risk modelling, see e.g. Jeanblanc and Rutkowski (2000), Bielecki and Rutkowski (2002), Duffie and Singleton (2003) and Lando (2004). We take a look at a very special case, that is maybe most useful when considering a stand-alone credit (instead of an entire credit portfolio) where statistical problems are difficult and one needs a simple robust method for pricing or comparing of different contracts. 
The approach we are going to present, is in the end based on martingale representation result for random measures. References for this topic are Chou and Meyer (1974), Jacod (1975) and Davis (1976). For a more systematic and quite general treatment of this approach see Leitner (2005).

Let the risk-free short interest rate $r \geq 0$ be a constant. Consider a single credit contract of the following type: At initial time $t=0$ an amount $B>0$ is paid out to the debtor. In return the lender is entitled to a fixed payment $M \geq 0$ at maturity time $0<T<\infty$ and a constant cash-stream $c d t$ over the time interval $[0, T]$. However, we do not exclude the possibility of default: There exists a stopping time $\tau>0$ describing the time of default, i.e. $\{\tau<\infty\}=\{\tau \leq T\}$ is the event of default and $\{\tau=\infty\}$ is the event of no default. For simplicity we assume $\tau$ to have strictly positive intensity over $[0, \tau \wedge T]$ and $P(\tau=\infty)>0$, i.e. default does not occur with probability 1 and it can occur with a strictly positive density at any time in $[0, T]$. Furthermore, we assume that if default occurs, the lender still receives a random recovery value $X M$ where $X$ is $[0,1]$ valued and independent of $\tau$, but no further cash-stream $c d t$ over the remaining time interval $[\tau, T]$. The filtration $\left(\mathcal{F}_{t}\right)_{t \geq 0}$ we are using is the internal filtration generated by the marked point process $\left(T_{i}, X_{i}\right)_{i \geq 1}$ where $T_{1}=\tau, X_{1}=X$ and $T_{i}=\infty, X_{i}=0$ for all $i \geq 2$, so that $\mathcal{F}_{\tau+t}=\mathcal{F}_{\tau}$ for all all $t \geq 0$ and $\mathcal{F}_{\tau_{-}}=\mathcal{F}_{0}$ is trivial. In other words, randomness enters our model only via the stopping time of the default event and the recovery rate.

The (non-discounted) cumulated cash-stream $Z^{C}$ for the lender is on $[0, \tau \wedge T$ ) given as

$$
Z_{t}^{C}=-B e^{r t}+\int_{0}^{t} c e^{r(t-s)} d s=-B e^{r t}+c \phi(t),
$$

where $\phi(t)=\frac{e^{r t}-1}{r}$ for all $t \geq 0$ if $r \neq 0$, resp. $\phi(t)=t$ for $r=0$. Hence the total balance of the credit contract at time $T$ for the lender is given as

$$
Z_{t}^{C}=-B e^{r T}+(c \phi(\tau)+X M) e^{r(T-\tau)} \mathbf{1}_{\{\tau \leq T\}}+(c \phi(T)+M) \mathbf{1}_{\{\tau=\infty\}} .
$$

Assume now, that instantaneous insurance contracts priced according to some fixed DPCP are available in the market. We are going to design an insurance contract that allows to determine $B$ from $r, c, M, \tau$ and $X$ by a self-financing exact off-setting argument. The contract is designed as follows: In return for a premium cash-stream $Y_{s} d s$, to be specified later, paid over $[0, \tau \wedge T]$, with accumulated value $V_{t}:=\int_{0}^{t} Y_{s} e^{r(t-s)} d s$, the insurer pays the amount $C_{\tau}=B e^{r \tau}-$ $c \phi(\tau)-X M+V_{\tau}$ to the lender in the default event $\{\tau<\infty\} . Y_{t}$ is calculated from $C_{t}$, the insurance payment given that default occurs at $t \in(0, T]$, according to the definition of the DPCP on $[0, \tau \wedge T)$. Note that on $\{t<\tau \wedge T\}$ the cost of insurance $V_{t}$ already payed over $[0, t)$ is a part the insured amount $C_{t}$. As we will see, this will facilitate exact off-setting considerably (in particular, compared to the case of a standard default swap with discrete time payments). The cumulated cash-stream $Z^{I}$ of the insurance contracts is given on $[0, T]$ as 


$$
Z^{I}=-V^{\tau}+C_{\tau} \mathbf{1}_{[\tau, T]} .
$$

Denote by $Z_{t}=Z_{t}^{C}+Z_{t}^{I}$ the lenders total (non-discounted) cumulated cash-stream generated by the combined credit and insurance contracts at time $t \in[0, T]$ (which is trivially self-financing). On $[0, \tau \wedge T)$ we then have $Z_{t}=-B e^{r t}+c \phi(t)-V_{t}$ and $Z_{t}=0$ on $[\tau, T]$ by definition of $C_{\tau}$. The total balance $Z_{T}$ at terminal time $T$ is thus given as

$$
\begin{aligned}
Z_{T} & =-B e^{r T}+\left(c \phi(\tau)+X M-V_{\tau}+C_{\tau}\right) e^{r(T-\tau)} \mathbf{1}_{\{\tau \leq T\}}+\left(c \phi(T)+M-V_{T}\right) \mathbf{1}_{\{\tau=\infty\}} \\
& =\left(-B e^{r T}+c \phi(T)+M-V_{T}\right) \mathbf{1}_{\{\tau=\infty\}} .
\end{aligned}
$$

Note that we can achieve (7) to hold for any $B$ and any DPCP. If we can achieve that in addition $V_{T}$ equals $c \phi(T)+M-B e^{r T}$ on $\{\tau=\infty\}$, then $Z_{T}$ vanishes a.s. and the insurance contract exactly off-sets the credit contract. Denote by $\tilde{Y}$, $\tilde{V}, \tilde{C}, \tilde{Z}^{C}, \tilde{Z}^{I}$ and $\tilde{Z}$ the corresponding processes resulting from replacing $B$ with $\tilde{B}>B$ in the definitions of $Y, V, C, Z^{C}, Z^{I}$ and $Z$ and assume $Z_{T}=\tilde{Z}_{T}=0$. If $\tilde{Y}_{t}>Y_{t}$ for $\tilde{C}_{t}>C_{t}$ holds on $[0, \tau \wedge T)$ then $\tilde{V}_{t}>V_{t}$ and $\tilde{Z}_{t}^{I}<Z_{t}^{I}$, hence $Z_{t}-\tilde{Z}_{t} \geq$ $(\tilde{B}-B) e^{r t}$ on $[0, \tau \wedge T)$. Now $P(\tau>T)>0$ implies $Z_{T}>\tilde{T}_{T}$ on $\{\tau>T\}$, contradicting our assumption. Furthermore, since the interest rate $r$ was assumed to be constant the market becomes deterministic over $(\tau, T]$ and since $\tau$ is assumed to have a strictly positive intensity over $[0, \tau \wedge T], Z_{T}=0$ can only be achieved if $C_{t}=B e^{r t}-c \phi(t)-X M+V_{t}$ holds on [0, $\left.\tau \wedge T\right]$. Therefore, given the insurance prices, i.e. in the market extended by instantaneous insurance contracts, in a very strong indifference sense, $-V_{0}^{C}=B$ is the only fair price (and in this sense it is the value in the extended market) of the credit contract (which is assumed to be an irreversible investment and can not be traded in any way) right after paying out $B$ to the borrower. This value found by the self-financing exact offsetting argument is risk-free in the sense, that at time $T$ it will not make a difference to the lender whether the credit contract was entered and $B$ was handed out or no transaction at all had been made. The lender makes a profit of $B-\tilde{B}$ iff $\tilde{B}<B$ is actually handed out to the borrower. This is a completely risk-free profit and not a profit over some indifference price and therefore independent of any preferences. Furthermore, on $\{t<\tau<\infty\},-Z_{t}$ is a risk-free price at time $t \geq 0$ for the defaultable credit and the remaining cash-stream over $[t, \tau \wedge T]$, since after receiving $-Z_{t}$ in exchange for all the rights the credit contract entitles to, the lender is back to the situation she would be in without having entered the credit contract in the first place.

3.1. Linear Case. We now use a DPCP as above in Example 2.2 in order to determine the insurance premium cash-stream $Y_{s} d s$ to be paid over $[0, \tau \wedge T]$. Assume that the recovery ratio $X$ is independent of $\tau$. Set $\tilde{\alpha}:=(1+\lambda) \alpha$ and $\gamma:=0$. We find the following expression for the premium density $Y_{t}$ on $[0, \tau]$, denoting by $K(t, \cdot)$ the kernel describing the conditional claim amount distribution of $C_{\tau}$ given $\tau=t$ : 


$$
\begin{aligned}
Y_{t} & =\alpha \mathcal{R}(K(t, \cdot)) \\
& =\tilde{\alpha} E\left[B e^{r t}-c \phi(t)-M X+V_{t}\right]+\tilde{\alpha} \tilde{\mathcal{R}}(-M X) \\
& =\tilde{\alpha} V_{t}+\tilde{\alpha}\left(B e^{r t}-c \phi(t)-M E[X]+\tilde{\mathcal{R}}(M \tilde{X})\right),
\end{aligned}
$$

where $\tilde{X}:=1-X$ is the fraction of loss. Note that on top of the losses caused by the default event, the accumulated insurance premiums are also covered by the insurance paying $C_{\tau}$ on $\{\tau<\infty\}$. In particular, the insurer can not use the already earned premiums in order to build up a reserve! The insurer gains only if no default occurs.

$$
\frac{d V_{t}}{d t}=Y_{t}+r V_{t} \text { leads to an inhomogeneous linear ODE for } V_{t} \text { : }
$$

$$
\frac{d V_{t}}{d t}=(\tilde{\alpha}+r) V_{t}+\tilde{\alpha}\left(B e^{r t}-c \phi(t)-M E[X]+\tilde{\mathcal{R}}(M \tilde{X})\right) .
$$

The variation-of-constants approach $V_{t}=W_{t} e^{(\tilde{\alpha}+r) t}$ leads to

$$
\frac{d W_{t}}{d t}=\tilde{\alpha}\left(B e^{r t}-c \phi(t)-M E[X]+\tilde{\mathcal{R}}(M \tilde{X})\right) e^{-(\tilde{\alpha}+r) t} .
$$

We find

$$
W_{t}=-\frac{\tilde{a}}{\tilde{\alpha}+r}\left(\frac{\tilde{a}+r}{\tilde{a}} B e^{r t}-c \phi(t)-\frac{c}{\tilde{a}} e^{r t}-M E[X]+\tilde{\mathcal{R}}(M \tilde{X})\right) e^{-(\tilde{\alpha}+r) t}+k,
$$

for a constant $k$, hence

$$
V_{t}=\left(\frac{c}{\tilde{a}+r}-B\right) e^{r t}+\frac{\tilde{\alpha}}{\tilde{\alpha}+r}(c \phi(t)+M E[X]-\tilde{R}(M \tilde{X}))+k e^{(\tilde{\alpha}+r) t} .
$$

Now the terminal condition $V_{T}=c \phi(T)+M-B e^{r T}$ implies

$$
k=\left(M-\frac{c}{\tilde{\alpha}+r}-\tilde{\alpha} \frac{M E[X]-\tilde{\mathcal{R}}(M \tilde{X})}{\tilde{\alpha}+r}\right) e^{-(\tilde{\alpha}+r) T} .
$$

We have $V_{0}=0$ iff

$$
B=M+(c-r M-\tilde{\alpha}(M E[\tilde{X}]+\tilde{\mathcal{R}}(M \tilde{X}))) \frac{1-e^{-(\tilde{\alpha}+r) T}}{\tilde{\alpha}+r} .
$$

Note that $B \leq M$ iff $c \leq r M+\alpha \mathcal{R}(M \tilde{X})$. We then find for the risk-free value of the defaultable credit $-Z_{t}$ at time $0 \leq t<\tau \wedge T$

$$
\begin{aligned}
-Z_{t} & =M+(c-r M-\tilde{\alpha}(M E[\tilde{X}]+\tilde{\mathcal{R}}(M \tilde{X}))) \frac{1-e^{-(\tilde{a}+r)(T-t)}}{\tilde{a}+r} . \\
& =M+(c-r M-\alpha \mathcal{R}(M \tilde{X})) \frac{1-e^{-(\tilde{a}+r)(T-t)}}{\tilde{a}+r} .
\end{aligned}
$$


Equation (10) provides the term structure of defaultable credits. In the case of a classical credit insurance, bought for an single initial payment at time $t=0$, and leading to the same result $Z_{T}=0$ as the DPCP-priced stream of insurances, it were not clear what a risk-free value for the credit contract should be at time $0<t<\tau \wedge T$. Furthermore, equation (10) allows risk-free pricing of modified contracts: Any combination of $c, M, \mathcal{R}$ that leads to the same value for $-Z_{t}$ defined by (10) at time $0<t<\tau \wedge T$ can be used for the modified contract, since the resulting $Z_{T}$ always vanishes. Contract modifications which lead to $-Z_{t}^{\prime}$ can be made risk-free value neutral by a payment of $Z_{t}^{\prime}-Z_{t}$ at time $t$.

It is not hard the check (see e.g. Bielecki and Rutkowski (2002), Chapter 8, and Leitner (2005)) that in the case $\tilde{R}(M \tilde{X})=0$, formula (10) corresponds to risk-neutral valuation under an EMM that changes the intensity of $\tau$ from $\alpha$ to $\tilde{\alpha}$ without changing the expectation of $X . \tilde{R}(M \tilde{X})$ is here to be interpreted as a non-linear risk premium charged as a compensation for the down-side risk due to a random loss fraction. In the case that $\tilde{\mathcal{R}}(M \tilde{X})=M E_{Q}[\tilde{X}-E[\tilde{X}]]$ for some probability measure $Q$ changing the law of $X$ without changing the law of $\tau$, we have $\mathcal{R}(M \tilde{X})=(1+\lambda)\left(M E[\tilde{X}]+M E_{Q}[\tilde{X}-E[\tilde{X}]]\right)=(1+\lambda) M E_{Q}[\tilde{X}]$. Formula (10) then corresponds to risk-neutral valuation under an EMM that changes the intensity of $\tau$ from $\alpha$ to $\tilde{\alpha}$ and the expectation of $\tilde{X}$ to $E_{Q}[\tilde{X}]$. If $\tilde{\mathcal{R}}$ is a coherent risk measure, $Q$ might depend on $X$. This implies the valuation principle to be non-linear in general. This can be interpreted in the following way: the choice of the pricing EMM depends on the contingent claim and can thus take down-side risk explicitly into account. However, our approach allows for self-financing replication in much more general situations, where down-side risk is priced by e.g. taking higher moments into account as well. We then have a truly non-linear valuation principle that can not be achieved by risk-neutral valuation.

Assume e.g. that $M$ equals the notional amount $B$ and assume $\tilde{\mathcal{R}}$ to be positive homogeneous. Setting $c=: \tilde{c} M$ we find by solving equation (9) the following expression for $\tilde{c}$ :

$$
\tilde{c}=r+(1+\lambda) E[\tilde{X}] \alpha+\tilde{\alpha} \tilde{\mathcal{R}}(\tilde{X})=r+\alpha \mathcal{R}(\tilde{X}) .
$$

This can be interpreted in the following way: In addition to the short risk free interest rate $r$, the borrower is charged a risk premium for expected losses of $(1+\lambda)$ times expected loss $E[\tilde{X}]$ times default intensity $\alpha$ and a risk premium $\tilde{\alpha} \tilde{\mathcal{R}}(\tilde{X})$ for so-called unexpected losses due to the volatility in the loss fraction $\tilde{X}$.

We can now calculate the claim amount $C_{t}$, given $\tau=t$ for $t \in[0, T]$. It turns out that $k=0$ holds and

$$
\begin{aligned}
C_{t} & =B e^{r t}-c \phi(t)-X M+V_{t} \\
& =\frac{c}{\tilde{a}+r}-X M+\frac{\tilde{\alpha}}{\tilde{\alpha}+r}(M E[X]-\tilde{R}(M \tilde{X}))=: M C, \\
& =\tilde{X} M+\frac{c-r M-\alpha \mathcal{R}(M \tilde{X})}{\tilde{a}+r}=: M C,
\end{aligned}
$$


where the distribution of $C>0$ does not depend on the time $t$. If the fact that the insurer can not build up a reserve from the premium cash-stream had caused a headache, we see now, that at least the insured amount and the premium density are constant.

Consider now the case $c=0$ : For the forward rate $r_{T}$ define by $B=M$ $\exp \left(-\int_{0}^{T} r_{s} d s\right)$ for all $T>0$, we then find

$$
r_{T}=\tilde{c}\left(1-\tilde{\alpha}(\tilde{\mathcal{R}}(\tilde{X})-E[X]) \frac{e^{(\tilde{\alpha}+r) T}-1}{\tilde{a}+r}\right)^{-1} .
$$

3.2. Super-Linear Case. We now use a DPCP as above with $\gamma>0$ in order to determine $V$. Assume that $X$ is independent of $\tau$. We find the following expression for the premium density $Y_{t}$ on $[0, \tau)$ :

$$
\begin{aligned}
Y_{t}= & \tilde{\alpha}\left(E\left[B-c \phi(t)-M X+V_{t}\right]+\tilde{\mathcal{R}}(M \tilde{X})\right)+\gamma E\left[\left(B-c \phi(t)-M X+V_{t}\right)^{2}\right] \\
= & \tilde{\alpha} V_{t}+\tilde{\alpha}(B-c \phi(t)-M E[X]+\tilde{\mathcal{R}}(M \tilde{X})) \\
& +\gamma\left(\left(B-c \phi(t)+V_{t}\right)^{2}-2\left(B-c \phi(t)+V_{t}\right) M E[X]+M^{2} E\left[X^{2}\right]\right) \\
= & \gamma V_{t}^{2}+V_{t}(\tilde{\alpha}+2 \gamma(B-c \phi(t)-M E[X]))+\tilde{\alpha}(B-c \phi(t)-M E[X]+\tilde{\mathcal{R}}(M \tilde{X})) \\
& +\gamma\left((B-c \phi(t))^{2}-2(B-c \phi(t)) M E[X]+M^{2} E\left[X^{2}\right]\right) \\
= & : \gamma V_{t}^{2}+g(t) V_{t}+h(t) .
\end{aligned}
$$

Again $\frac{d V_{t}}{d t}=Y_{t}+r V_{t}$ leads to a ODE for $V_{t}$, this time of Riccati type:

$$
\frac{d V_{t}}{d t}=\gamma V_{t}^{2}+(g(t)+r) V_{t}+h(t)=\left(\sqrt{\gamma} V_{t}+\frac{g(t)+r}{2 \sqrt{\gamma}}\right)^{2}+h(t)-\frac{(g(t)+r)^{2}}{4 \gamma} .
$$

With the transformation $W_{t}:=V_{t} \gamma+\frac{g(t)}{2}$ we reach the following normal form of a Riccati ODE:

$$
W_{t}^{\prime}=V_{t}^{\prime} \gamma+\frac{g^{\prime}(t)}{2}=W_{t}^{2}+\gamma h(t)-\frac{(g(t)+r)^{2}}{4}+\frac{g^{\prime}(t)}{2} .
$$

In our special case, it turns out that $K:=W_{t}^{2}-W_{t}^{\prime}$ is actually constant: We find

$$
K=\frac{\tilde{\alpha}^{2}}{4}+\gamma(c-\tilde{\alpha} \tilde{\mathcal{R}}(M \tilde{X}))-\gamma^{2} M^{2} \operatorname{Var}(X)+r\left(\frac{\tilde{a}^{2}}{2}+\gamma(B-M E[X])\right) .
$$

In particular, we are in the lucky position to be able to solve the Riccati ODE (14) explicitly. Assume $\gamma>0$ to be chosen small enough so that $b^{2}:=K>0$ holds. Separating variables and integrating $\left(W^{2}-b^{2}\right)^{-1} d W=d t$, we find $W_{t}=$ $b \frac{1+\exp (2 b(k+t))}{1-\exp (2 b(k+t))}$ for some constant $k$. Choosing $k=(2 b)^{-1} \log \left(\frac{w-b}{w+b}\right)-T$ for a constant $w>b$ we have $W_{T}=w$ and for all $0 \leq t \leq T$ 


$$
W_{t}=r \frac{(w+b)+(w-b) \exp (2 b(t-T))}{(w+b)-(w-b) \exp (2 b(t-T))} .
$$

Furthermore, $W_{0}=x$ is equivalent to

$$
w=-b \frac{(b-x)-(x+b) e^{-2 b T}}{(b-x)+(x+b) e^{-2 b T}} .
$$

Since $V_{T}=c T+M-B$ iff $W_{T}=w:=\gamma(c T+M-B)-\frac{g(T)}{2}=2 \gamma(c T-B+$ $\left.M \frac{1+E[X]}{2}\right)-\frac{\tilde{a}}{2}$ and $W_{0}=V_{0} \gamma-\frac{g(0)}{2}$ we find $V_{0}=0$ iff $W_{0}=-\frac{g(0)}{2}$, hence $V_{0}=0$ iff the following equation holds with $x:=-\frac{g(0)}{2}=\gamma(M E[X]-B)-\frac{\tilde{a}}{2}$ :

$$
2 \gamma\left(c T-B+M \frac{1+E[X]}{2}\right)-\frac{\tilde{a}}{2}=-r \frac{(r-x)-(x+r) e^{-2 r T}}{(r-x)+(x+r) e^{-2 r T}},
$$

from which quadratic equations for $B$ resp. $M$ or a equation for $c$ is easily derived.

\section{ACKNOWLEDGMENTS}

I am grateful to an anonymous referee for helpful remarks and suggestions.

\section{REFERENCES}

Artzner, P., Delbaen, F., Eber, J.-M. and Heath, D. (1999) Coherent Measures of Risk. Mathematical Finance 9, 203-228.

Bielecki, T. and Rutkowski, M. (2002) Credit Risk: Modeling, Valuation and Hedging. Springer Finance, Vol. 89. Springer: Berlin.

Brémaud, P. (1981) Point processes and queues, martingale dynamics. Springer Series in Statistics. Springer: New York.

BRÉmAud, P. and JACOD, J. (1977) Processus ponctuels et martingales: Résultats récents sur la modélisation et le filtrage. Advances in Applied Probability 9, 362--416.

Chou, C.-S. and Meyer, P.A. (1974) La représentation des martingales relatives à un processus punctuel discret. Comptes Rendus de l'Académie des Sciences de Paris, Série A278, 1561-1563.

DAVIS, M.H.A. (1976) The Representation of Martingales of Jump Processes. SIAM Journal of Control and Optimization 14(4), 623-638.

Delbaen, F. and Schachermayer, W. (1994) A general version of the fundamental theorem of asset pricing. Mathematische Annalen 300, 463-520.

Delbaen, F., Monat, P., Schachermayer, W., Schweizer, M. and Stricker, C. (1997) Weighted norm inequalities and hedging in incomplete markets. Finance and Stochastics 1, 181-227.

Delbaen, F., Grandits, P., Rheinländer, T., SAmperi, D., Schweizer, M. and Stricker, C. (2002) Exponential hedging and entropic penalties. Mathematical Finance 12, 99-123.

Duffie, D. and Singleton, K. (2003) Credit Risk: Pricing, Measurement and Management. Princeton University Press: Princeton and Oxford.

Föllmer, H. and Schied, A. (2002) Stochastic Finance. Berlin: de Gruyter.

FritTELLI, M. (2000) The minimal entropy martingale measure and the valuation problem in incomplete markets. Mathematical Finance 10, 39-52.

Gerber, H.U. (1979) An Introduction to Mathematical Risk Theory. Huebner Foundation Monographs. University of Pennsylvania. 
Goovaerts, M., KaAs, R., Dhaene, J. and TAng, Q. (2003) A unified approach to generate risk measures. ASTIN Bulletin, 33(2), 173-191.

JACOD, J. (1975) Multivariate Point Processes: Predictable Projection, Radon-Nikodym Derivatives, Representation of Martingales. Zeitschrift für Wahrscheinlichkeitstheorie und verwandte Gebiete 31, 235-253.

Jacod, J. and Shiryaev, A.N. (1987) Limit Theorems for Stochastic Processes. Springer: Berlin.

Jeanblanc, M. and Rutkowski, M. (2000) Modelling of default risk: an overview. In: Mathematical Finance: Theory and Practice, p. 171-269. Higher Education Press: Beijing.

Kramkov, D. and Schachermayer, W. (1999) The asymptotic elasticity of utility functions and optimal investment in incomplete markets. Annals of Applied Probability 9(3), 904-950.

LAndo, D. (2004) Cedit Risk Modelling: Theory and Applications. Princeton University Press: Princeton, New Jersey.

Last, G. and Brandt, A. (1995) Marked Point Processes on the Real Line. Springer: Berlin.

LEITNER, J. (2005) Event Insurance Markets. Submitted.

LiptSer, R. and Shiryaev, A.N. (2000) Statistics of random processes II. Applications. Applications of Mathematics. Stochastic modelling and applied probability 6. Springer: Berlin.

Miкоsch, T. (2004) Non-Life Insurance Mathematics. Springer: Berlin.

SCHACHERMAYER, W. (2001) Optimal investment in incomplete markets when wealth may become negative. Annals of Applied Probability 11(3), 694-734.

WANG, S. (1996) Premium Calculation by Transforming the Layer Premium Density. ASTIN Bulletin 26(1), 71-92.

\section{JOHANNES LEITNER}

Research Unit for Financial and Actuarial Mathematics,

Institute for Mathematical Methods in Economics,

Vienna University of Technology,

Wiedner Hauptstraße 8-10/105,

A-1040 Vienna, Austria.

E-mail: jleitner@fam.tuwien.ac.at 